\title{
La persévérance chez les enseignants franco-ontariens ${ }^{1}$
}

\author{
Louise M. Bélair, UQTR \\ Christine Lebel, UQTR
}

\begin{abstract}
Résumé
Cet article examine divers phénomènes ayant permis à des enseignants de trouver du sens à leur profession et persévérer dans l'exercice de celle-ci, notamment dans les premières années d'entrée en fonction. Plus précisément, notre recherche porte sur une dimension peu explorée dans la littérature, et s'attarde à des éléments positifs marquants de la profession relatés par des enseignants franco-ontariens. Ces épisodes de récits de vie racontées de manière anecdotique procèdent d'une forme privilégiée d'expression tant de la formation et de l'identité des personnes que de la quête de sens, en rapport avec le vécu subjectif des enseignants, dans un contexte minoritaire. La question générale de cette étude se présente comme suit : Quels sont, d'après les enseignants franco-ontariens, les événements saillants positifs qui les ont marqués surtout en début de carrière, et qui favorisent chez eux la persévérance dans l'exercice de la profession ? Les résultats sont discutés à partir de trois préoccupations: les catégories d'évènements marquants positifs qui émergent ; les différences relatives au profil de carrière selon le modèle de Huberman (1989) ; les différences relatives au sexe et à l'expérience.
\end{abstract}

\begin{abstract}
This article examines various phenomena that allow teachers to find a meaning in their profession and persevere in the discharge of their duties, especially during their first years in this field. More precisely, our research focuses on a less explored dimension in the literature and dwells upon the profession's most significant positive elements reported by Franco-Ontarian teachers. These life stories told in an anecdotal manner, come from a privileged form of expression as much from their training and from their personal identity as well as their quest for meaning, dealing with the teachers' subjective real-life experiences in a minority context. The general question of this study is: According to these FrancoOntarian teachers, what are the most prominent and positive events that have influenced them, especially during the beginning of their career, and that have furthered in them perseverance in the discharge of their duties? We then discuss the findings from the three preoccupations: emerging positive events, differences related to their career's profile, according to Huberman's model (1989); and differences related to their gender and experience.
\end{abstract}




\section{Problématique et contexte de la recherche}

À l'instar de plusieurs pays industrialisés et provinces canadiennes, les écoles francophones de l'Ontario font face à une pénurie d'enseignants, tant au primaire qu'au secondaire depuis plus de deux décennies. L'augmentation du nombre de lettres de permission en fait foi ${ }^{2}$, dans certaines régions plus éloignées, et la demande en personnel franco-ontarien compétent et qualifié ne cesse de croître.

Une faction non négligeable d'étudiants diplômés ${ }^{3}$ récemment, occupant un emploi dans les écoles francophones primaires ou secondaires de l'Ontario, quittent effectivement l'enseignement dans les deux premières années (Ordre des enseignantes et enseignants de l'Ontario, 2003a et b). Des statistiques québécoises vont dans le même sens en démontrant un taux d'abandon oscillant autour des $4 \%$ parmi les jeunes enseignants durant les premières années d'entrée en fonction et de $20 \%$ dans l'ensemble du corps professoral (Mukamurera, 2006). Les raisons invoquées par ces nouveaux enseignants sont principalement liées à la lourdeur de la tâche, aux groupes-classes difficiles et à la précarité de l'emploi (Mukamurera, 2006). Lessard et Tardif (2003, p. 13) abondent d'ailleurs en ce sens lorsqu'ils affirment : «...(qu') une large part de l'évolution concrète de leur métier se trame au jour le jour dans leur rapport émotionnels et normatifs aux élèves».

Ces douze à trente-six premiers mois d'insertion professionnelle, qualifiés par Huberman (1989) d'années de tâtonnement et de survie seraient ainsi particulièrement cruciaux en ce qui a trait à la décision de poursuivre ou non la carrière en enseignement. Or, les besoins accrus de personnel enseignant francophone en milieu minoritaire, auxquels s'ajoute la problématique de la désertion scolaire au sein du corps enseignant en général, font en sorte qu'il convient d'une part de réfléchir à la construction d'un répertoire d'outils riches et variés aptes à freiner cette tendance. D'autre part, il importe également de considérer la mise en place de dispositifs de formation susceptibles de permettre aux futurs enseignants de relever de manière pertinente les nombreux défis rattachés à la pratique de l'enseignement au $21^{\text {ème }}$ siècle et de favoriser le développement d'aptitudes propres à la persévérance.

Des récits de pratiques portant sur des évènements positifs marquants dans la carrière, ont été recueillis auprès d'enseignants en exercice, en vue de répondre à la question suivante: Quels sont, d'après les enseignants francoontariens, les événements saillants positifs qui les ont marqués surtout en début de carrière, et qui favorisent chez eux la persévérance dans l'exercice de la profession?

\section{Cadre conceptuel proposé pour expliquer la persévérance en enseignement.}

La littérature scientifique abonde de recherches menées sur la persévérance, particulièrement dans les études et en fonction de la réussite scolaire, mais ces dernières définissent très peu de manière conceptuelle la notion de persévérance. Les travaux de Csikszentmihalyi (1990) montrent qu'une personne persévérante est dotée d'un ensemble d'attitudes, de valeurs et de savoir-être qui, mis ensemble, font qu'elle persévère. La difficulté réside cependant dans le fait que le modèle d'intégration de ces valeurs et attitudes n'est pas unique, voire même individuel. 


\section{Les éléments constitutifs de la persévérance}

Jusqu'à ce jour, nos recherches nous ont permis de cerner différents concepts qui semblent intervenir dans l'action de persévérer. Un schéma de référence initial a été élaboré à cet égard afin de nous permettre de mieux comprendre comment chacun de concepts contribue à alimenter cette action chez des individus. Ce schéma qui sert de base dans la présentation de ce cadre n'est cependant pas définitif; nos recherches en cours permettront de peaufiner les relations qu'entretiennent ces concepts entre eux. Trois regroupements sans primauté de l'un sur l'autre, se dessinent donc : le sentiment d'auto-efficacité, la résilience et le plaisir.

Figure 1. Illustration d'un cadre de référence sur la persévérance

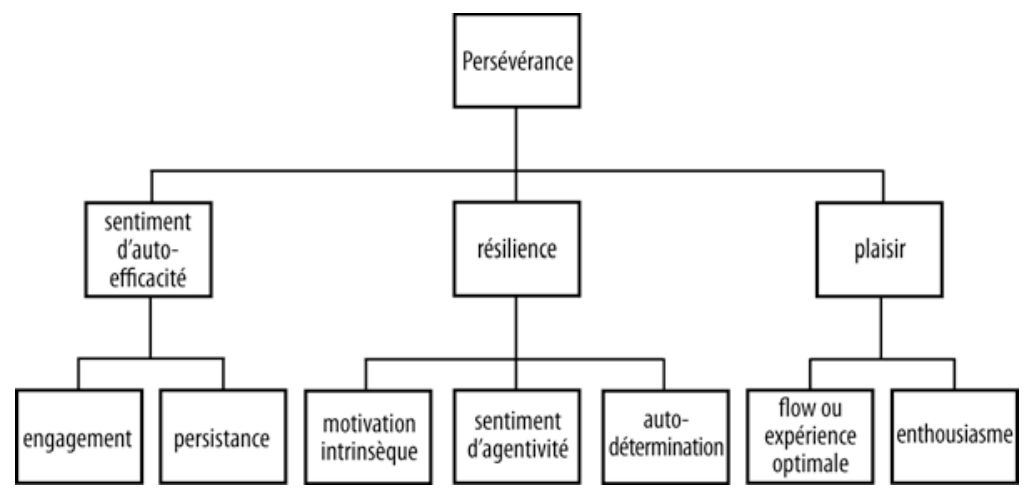

\section{Le sentiment d'auto-efficacité}

Le sentiment d'auto-efficacité (Bandura, 2001) se traduit par la capacité d'une personne à organiser et à exécuter les actions pour gérer des situations données. C'est également la croyance que la personne d'être en mesure de produire un effet et d'agir sur son environnement. L'efficacité personnelle perçue concerne ainsi la croyance de l'individu en sa capacité d'exécuter la ligne de conduite requise pour produire des résultats souhaités. Cette composante du cadre théorique a été identifiée comme un élément central dans le contexte de la pratique enseignante. De fait, elle semble être le résultat de deux facteurs mentionnés dans les diverses études : l'engagement et la persistance.

\section{L'engagement}

L'engagement est défini comme étant une décision qui influe clairement sur les comportements ultérieurs (Festinger, 1964), une position qui n'est pas facile à abandonner, un choix irrévocable (Secord et Backman, 1974). Il se traduit par la promesse ou l'obligation de maintenir une ligne d'action (Kiesler, 1971). À titre d'exemple, l'engagement pourrait s'illustrer chez un enseignant qui, malgré les difficultés de faire converser ses élèves en français, persiste et propose des activités d'improvisation pour rendre cette langue encore plus attrayante auprès de ces derniers. 


\section{La persistance}

Le concept de persistance est souvent associé à celui de la motivation à poursuivre une tâche complexe. Étudié surtout dans le domaine de la psychologie, où il est défini comme une caractéristique importante du comportement intentionnel (Hebb, 1949), il s'apparente selon Bourdages (2001) à une résolution de problème complexe où l'individu confronté au choix de poursuivre dans la tâche non résolue ou d'entreprendre une tâche alternative, décide de persister dans cette tâche.

\section{La résilience}

Issue de la physique, la théorie de la résilience a été particulièrement popularisée par Cyrulnik (1999) alors qu'il tentait de comprendre la capacité qu'ont des individus à faire face à des situations conflictuelles ou adverses et pourquoi certaines personnes sont capables de rebondir après les épreuves alors que d'autres y arrivent difficilement. La résilience semble s'accompagner d'attitudes relatives à trois concepts forts importants: la motivation intrinsèque et l'autodétermination ainsi que le sentiment d'agentivité.

\section{La motivation intrinsèque et l'autodétermination}

La motivation est un concept utilisé pour décrire les forces internes et/ou externes produisant le déclenchement, la direction, l'intensité, et la persistance du comportement (Legendre, 1993). L'équipe de Deci et Ryan (1985) soutient qu'en termes théoriques, il existe divers types de motivation allant de l'extrinsèque à l'intrinsèque, pouvant s'articuler en cohérence avec le locus de causalité perçu (la source du contrôle de la motivation) et le processus d'autorégulation des actions. Dans cet ordre d'idée, la motivation intrinsèque permet à la personne de s'autodéterminer (via l'autorégulation) dans l'action et de trouver un sens aux situations difficiles, au point de persévérer.

\section{Le sentiment d'agentivité}

Le sentiment d'agentivité humaine (Bandura, 2001) est un concept qui se caractérise par la capacité d'exercer un contrôle sur les processus de sa pensée, sur sa motivation et ses actions. L'agentivité émergente se retrouve chez des individus qui font des contributions causales de leur propre action et motivation dans un rapport triadique entre les facteurs personnels, comportementaux et environnementaux. Elle est selon Bandura (1986) directement en rapport avec la théorie sociale cognitive au sens où une personne démontrant de l'agentivité, agit comme un agent qui influence sa façon de fonctionner et les évènements qui affectent sa vie. «Dans cette vision, les enseignants sont les contributeurs actifs à leurs circonstances de vie et non des produits des circonstances» (Montclerc, 2006).

\section{Le plaisir}

Le plaisir, fréquemment associé au bonheur (Myers et Diener, 1997), est intimement lié aux notions de contrôle, d'autodétermination et de persistance. De fait, Baumeister (1991) cité par Argyle (1997) identifie quatre besoins essentiels pour donner un sens à la vie d'un individu: 1) Avoir un projet de vie; 2) Avoir un 
ensemble de valeurs de base; 3) Le sentiment d'avoir un contrôle sur les événements; 4) Avoir le sens de la valeur personnelle. (p. 105). Les tenants de la recherche positive (Seligman et Csikszentmihalyi) mettent également le plaisir en lien avec l'expérience optimale et avec l'enthousiasme.(Heutte, 2006).

\section{Le flow ou l'expérience optimale}

Le concept de flow ou l'expérience optimale Csikszentmihalyi (1990). se caractérise par un état dans lequel les individus sont tellement impliqués dans ce qu'ils font que plus rien d'autre ne compte. La satisfaction que produit ce genre d'activité est telle, que les personnes sont prêtes à les faire sans penser à ce qu'elles pourront en tirer et cela même lorsque les situations sont difficiles ou dangereuses. Le flow peut ainsi être une façon d'expliquer l'état d'une personne qui se retrouve dans des situations de défis qui lui permettent d'exploiter à fond son niveau de compétence, en autant qu'il existe un niveau d'équilibre entre le défi à relever et le sentiment de compétence de la personne. Selon Singer, Hausenblas et Janelle (2001), faire l'expérience du flow reflète l'absence de stress, d'anxiété ou d'ennui, ce qui occasionne un sentiment d'ivresse, permettant de décrire l'expérience optimale. Cet état entraînerait alors la sensation d'exercer un contrôle, sorte de fusion entre l'action et la conscience permettant d'accéder à une expérience auto-satisfaisante (Csikzentmihalyi, 1990). Autrement dit, faire l'expérience du flow dans la pratique enseignante consisterait à être complètement absorbé par son travail, uniquement pour le plaisir de le faire. L'enseignant serait entièrement impliqué dans son activité et utiliserait ses compétences à leur plus haut niveau.

\section{L'enthousiasme et la passion}

L'enthousiasme et la passion sont des composantes affectives qui font en sorte qu'une personne choisit un objet social, perçu comme attirant ou valorisé parmi plusieurs autres. Ces composantes affectives peuvent être comparées à des pouvoirs d'attraction qui mènent une personne à s'investir pour une cause en particulier. Selon Dubé, Kairouz et Jodoin (1997), la personne qui éprouve de l'enthousiasme et de la passion donne son attention, son énergie et son temps à un objet social parce que «ses actions lui procurent de la joie et des récompenses » (p.218). Dans la pratique enseignante, l'enthousiasme et la passion se traduiraient alors en un enjouement pour son travail d'interaction avec ses élèves, lequel serait valorisant socialement. C'est dans cette perspective que l'on peut supposer que la persévérance a comme précurseurs l'enthousiasme et la passion, puisque ces composantes affectives alimenteraient la persévérance dans des contextes difficiles.

Ce cadre théorique ouvre sur une méthodologie de recherche permettant de saisir le sens que les enseignants donnent à leur expérience, les éléments qu'ils choisissent de dévoiler et comment ils en parlent. Cette méthode qui est susceptible de faciliter l'émergence de concepts qui n'ont pas été apparents dans la littérature jusqu'à présent, tente de mettre en exergue ce qui est signifiant dans le vécu des personnes et de voir comment cette signification est articulée dans leur esprit. 


\section{Méthodologie}

L'approche narrative (Malrieu, 2003) a été utilisée en vue de faire émerger un ensemble de facteurs qui participent de l'adhésion et de la persévérance dans la profession. L'analyse des données qui s'en est suivie a permis de mettre en évidence un ensemble d'éléments qui ont été catégorisés à la lumière d'une revue de littérature sur cette problématique.

\section{Participantes et participants au projet}

Le nombre total de participants à cette phase de la recherche (janvier à décembre 2005) est de 40 enseignants franco-ontariens des régions d'Ottawa, de Hawkesbury et de Toronto, dont 12 proviennent du secondaire et 28 du primaire. Dans l'optique d'une analyse rigoureuse du phénomène de la persévérance, ces personnes ont été classifiées en fonction de la catégorisation des étapes de vie professionnelle proposée par Huberman (1989). Ces catégories sont présentées ici, avec le nombre d'hommes et de femmes ayant participé à cette recherche.

Tableau 1.

Répartition des participants selon les années d'expérience et le sexe

\begin{tabular}{|l|c|c|c|}
\hline \multicolumn{1}{|c|}{ Années d'expériences } & Hommes & Femmes & Total \\
\hline Entre 1 et 3 ans «le tâtonnement» & 3 & 6 & 9 \\
\hline Entre 4 et 10 ans «la consolidation» & 7 & 8 & 15 \\
\hline $\begin{array}{l}\text { Entre } 11 \text { et } 20 \text { ans «diversification et } \\
\text { remise en question» }\end{array}$ & 2 & 9 & 11 \\
\hline $\begin{array}{l}20 \text { ans et plus «sérénité et } \\
\text { conservatisme» }\end{array}$ & 1 & 4 & 5 \\
\hline Total & 13 & 27 & $\mathbf{4 0}$ \\
\hline
\end{tabular}

\section{Outils de collecte de données.}

Inspirée des modèles de collecte de données axés sur la narration et plus particulièrement, sur la narration de récits de pratiques de type biographique (Lani-Bayle et Bire, 2003 ; Olivier et Demouge, 1999), notre approche a consisté à demander à des enseignants de se souvenir d'un événement marquant et de le reconstituer en le racontant par écrit ou oralement. Puisque seulement 8 des 40 enseignants ont choisi de rédiger par écrit leur histoire, un protocole d'entretien permettant de faire émerger les aspects positifs de la profession et de mieux comprendre les facteurs inhérents à la persévérance dans l'enseignement, a été élaboré en fonction de deux grandes questions : Y a-t-il un événement marquant positif que vous aimeriez raconter? Il est peut-être arrivé à un moment donné, d'avoir envie de tout laisser tomber - alors pourquoi êtes-vous resté ?

\section{Analyse et interprétation}

Cette section présente les éléments les plus récurrents selon les groupes de participants, ainsi que l'analyse et l'interprétation des récits de pratiques. 


\section{Les éléments issus des analyses et leurs référents théoriques}

Afin cerner les paramètres contenus dans les diverses histoires, la première démarche d'analyse a consisté en l'identification puis en la validation inter-juges des facteurs phares liés à la persévérance de ces enseignants. Les concepts issus de la littérature qui se rattachent aux facteurs, sont indiqués en italique.

1. Les yeux des élèves qui s'allument (l'élève qui comprend tout à coup) autoefficacité

2. Hé! Salut Monsieur (la reconnaissance des élèves, immédiate ou dans l'après-coup)

3. C'est toujours vivant et varié (absence de monotonie)

4. Ah! La collaboration de cette collègue (coopération) engagement

5. Une maman m’a écrit «bravo!» (reconnaissance des parents)

6. J'apprends tout le temps (apprentissage continuel ) expérience vicariante

7. Je sais que je peux faire la différence (changer des choses dans la vie des élèves) agentivité

8. La tape dans le dos de la direction (reconnaissance et appui de la direction)

9. Et toute la classe a ri ensemble (complicité avec les élèves) plaisir

10. Je bâtis des projets pédagogiques avec les élèves (travail enseignant) motivation intrinsèque et autodétermination.

\section{Les récurrences chez les participants}

Les facteurs identifiés de manière récurrente par les participants, sont présentés en fonction de l'expérience de vie professionnelle, du niveau d'enseignement et du sexe.

Tableau 2.

Synthèse des analyses des entretiens et des histoires, pour les 1 à 3 ans d'expérience.

\begin{tabular}{|c|c|c|}
\hline Années d’expérience & Éléments récurrents (du + au -) & Nb.d'extraits \\
\hline 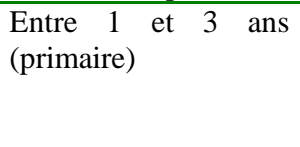 & $\begin{array}{l}\text { Les yeux qui s'allument } \\
\text { Vivant et varié } \\
\text { Collaboration avec les collègues } \\
\text { J'apprends tout le temps }\end{array}$ & $\begin{array}{l}7 \\
2 \\
2 \\
2\end{array}$ \\
\hline $\begin{array}{l}\begin{array}{l}\text { Entre } 1 \text { et } 3 \\
\text { (secondaire) }\end{array} \\
\end{array}$ & Faire la différence & $1 / 1$ \\
\hline $\begin{array}{l}\begin{array}{l}\text { Entre } 1 \\
\text { (femmes) }\end{array} \\
\text { et }\end{array}$ & $\begin{array}{l}\text { Les yeux qui s'allument } \\
\text { Collaboration avec les collègues } \\
\text { J'apprends tout le temps }\end{array}$ & $\begin{array}{l}5 \\
2 \\
1\end{array}$ \\
\hline $\begin{array}{l}\begin{array}{l}\text { Entre } 1 \\
\text { (hommes) }\end{array} \\
\text { et } 3 \text { ans }\end{array}$ & $\begin{array}{l}\text { Les yeux qui s'allument } \\
\text { Vivant et varié } \\
\text { J'apprends tout le temps } \\
\text { Faire la différence }\end{array}$ & $\begin{array}{l}2 \\
2 \\
1 \\
1\end{array}$ \\
\hline
\end{tabular}




\section{a. Les années de tâtonnement.}

Le tableau 2 présente les résultats pour le groupe des 1 à 3 ans d'expérience et ce, en divisant les personnes oeuvrant au primaire et au secondaire puis, les hommes des femmes.

Ces premières années, appelées «tâtonnement» par Huberman (1989) devraient de fait, prendre certaines colorations particulières. L'enseignant novice, est en mode de survie et au stade de la découverte. Il tâte le terrain, expérimente, ose, et surtout il est confronté à ce qu’Huberman nomme : «le choc du réel» puisqu'il découvre la nature du métier et se doit de réajuster ses idéaux de pratique à une réalité parfois moins sereine que ce qu'il croyait au moment de sa formation. Ces constats souvent douloureux, amènent effectivement ces nouveaux enseignants à privilégier des stratégies de survie qui revêtent diverses formes. Rayou et Van Zanten (2004), montrent, au terme d'une vaste enquête auprès de nouveaux enseignants oeuvrant dans des établissements d'enseignement difficiles en France, que certains d'entre eux optent pour quitter l'enseignement, d'autres s'orientent vers des établissements où les clientèles paraissent moins difficiles et qu'une autre faction choisit de considérer que «l'exercice même du métier est tout autant pourvoyeur de solutions que de problèmes » (p. 70).

Pour ces nouveaux enseignants, les défis à relever sont d'autant plus intéressants que «par-delà leur aspect d'accomplissement personnel, ils permettent de progresser véritablement dans le métier et de concilier son intérêt propre et celui des élèves » (ibid, p. 70). Se profile donc une forme d'authenticité chez ces nouveaux enseignants, où l'idée de " vocation » traduite par des éléments qui s’y rapprochent et le plaisir éprouvé, participent de cette capacité de faire face à la situation (ibid.). Tel que mentionné précédemment, les recherches en psychologie positive (Seligman, Csikszentmihalyi), parlent pour leur part d'enthousiasme et de passion, et mentionnent que ces deux éléments peuvent être

porteurs du projet de persévérer. À la lecture du tableau 2, on remarque d'emblée que la plupart des participants, tous du primaire sauf un seul du secondaire, qu'ils soient hommes ou femmes, misent surtout sur la classe et tout particulièrement sur les élèves, et indiquent que ce qui les fait persévérer, c'est de voir que les élèves ont compris, qu’ils réussissent, qu'ils se relèvent de leurs difficultés :

$A u$ jardin, on a souvent des élèves qui sont anglophones et j'en avais un qui est arrivé, il y a deux ans, et qui ne connaissait quasiment rien en français. Il connaissait peut-être "bonjour». Et c'est juste de le voir, d'interagir avec lui tous les jours et de voir comment il se développait. Et en trois mois, de septembre à décembre, tu voyais comment cet élève-là avait déjà appris la langue. Tu te sens fière de l'avoir aidé, de lui avoir permis de s'exprimer et de se pratiquer. Ça c'était un de mes gros moments parce que cet enfant-là avait beaucoup de difficultés et juste de le voir déboucher, c'était incroyable. Dans trois ans, je n'ai pas eu beaucoup d'expériences, mais celle-là m'a vraiment touchée.

L'expérience de maîtrise, donc le sentiment d'autoefficacité, serait effectivement essentiel au novice dans la profession (Bandura, 1986). 
Deux autres éléments sont également privilégiés par un bon nombre de participants. Il s'agit de l'aspect vivant et varié, soulevé par des hommes, et la collaboration avec les collègues notamment des enseignants plus expérimentés qui font parfois office de mentors. Cette collaboration est vécue comme un appui, une «bouée de sauvetage», ou un appel à l'aide entendu par un autre collègue. Nombre de recherches portant sur le mentorat, insistent sur l'importance de l'accompagnement par les pairs dans l'entrée dans le métier (Philion, 2005), mais indiquent que ce mentorat ne doit pas être obligatoire, ni imposé. Les enseignants débutants ou plus expérimentés, veulent effectivement pouvoir choisir librement, au gré des affinités, des goûts et de leurs préoccupations du moment, leurs collaborations, voire leurs mentors (Rayou et Van Zanten 2004). Le pattern qui s'esquisse pour ces débutants peut être présenté comme suit :

les moments qui favorisent la persévérance semblent se vivre au quotidien, dans la salle de classe, et la collaboration avec les collègues semble indispensable pour aider à passer au travers les difficultés rencontrées dans l'apprentissage de ce nouveau métier. Ces enseignants aiment aussi le fait que ce travail est varié et vivant, tandis que les enseignantes semblent trouver que leur propre apprentissage est aidant pour mieux comprendre ce métier.

Enfin, le seul enseignant du secondaire entre 1 et 3 ans d'expérience, a signifié que l'élément le plus important pour lui était de pouvoir faire la différence auprès des élèves; facteur qui s'inscrit au plan de l'autoefficacité et de l'agentivité.

\section{b. Les années de consolidation}

Tableau 3.

Synthèse des analyses des entretiens et des histoires, pour les 4 à 10 ans d'expérience.

\begin{tabular}{|c|c|c|}
\hline Années d’expérience & Éléments récurrents (du + au -) & Nb. d'extraits \\
\hline  & $\begin{array}{l}\text { Les yeux qui s’allument } \\
\text { Collaboration avec les collègues } \\
\text { Reconnaissance des élèves } \\
\text { Complicité }\end{array}$ & $\begin{array}{l}4 \\
4 \\
2 \\
2\end{array}$ \\
\hline $\begin{array}{l}\text { Entre } 4 \text { et } 10 \text { ans } \\
\text { (secondaire) }\end{array}$ & $\begin{array}{l}\text { Collaboration avec les collègues } \\
\text { Les yeux qui s'allument } \\
\text { Reconnaissance des élèves } \\
\text { Vivant et varié ; } \\
\text { Reconnaissance des parents } \\
\text { J’apprends tout le temps } \\
\text { Les projets }\end{array}$ & $\begin{array}{l}5 \\
4 \\
3 \\
2 \\
2 \\
2 \\
2\end{array}$ \\
\hline $\begin{array}{llll}\begin{array}{l}\text { Entre } 4 \\
\text { (femmes) }\end{array} & \text { et } & 10 & \text { ans } \\
\end{array}$ & $\begin{array}{l}\text { Les yeux qui s'allument } \\
\text { Collaboration avec les collègues } \\
\text { Reconnaissance des élèves }\end{array}$ & $\begin{array}{l}7 \\
7 \\
2\end{array}$ \\
\hline $\begin{array}{llll}\begin{array}{l}\text { Entre } 4 \\
\text { (hommes) }\end{array} & \text { et } & 10 & \text { ans } \\
\end{array}$ & $\begin{array}{l}\text { Reconnaissance des élèves } \\
\text { Collaboration avec les collègues } \\
\text { J'apprends tout le temps } \\
\text { Les projets }\end{array}$ & $\begin{array}{l}3 \\
2 \\
2 \\
2\end{array}$ \\
\hline
\end{tabular}


Les 14 enseignants de 4 à 10 ans s'insèrent dans ce que Huberbam, 1989, nomme la phase de stabilisation et de consolidation d'un répertoire pédagogique, laquelle fait suite à la phase initiale d'exploration. C'est à ce stade que l'enseignant sent qu'il appartient de plus en plus à une profession, qu'il réalise qu'il a un rôle essentiel à jouer dans l'éducation. Le sentiment d'efficacité qu'il développe, se réalise avec son groupe d'appartenance, d'où le concept d'efficacité collective amené par Bandura (2003). Ce sentiment lui permet de percevoir une plus grande maîtrise de ses compétences professionnelles, et favorise le développement d'un sentiment d'efficacité personnelle par une confiance envers ses propres capacités. Le tableau 3, montre cette tendance. On voit apparaître de nouveaux éléments soit la reconnaissance, la complicité et les projets. On observe que l'accent est encore mis sur les élèves, mais cette vision procède d'un élargissement qui englobe désormais, à la fois l'école et l'extérieur de l'école.

Certaines divergences entre les participants du primaire et du secondaire, de même qu'entre les femmes et les hommes s'observent ici. Chez les enseignants du primaire, c'est d'abord et avant tout la classe (les yeux), suivie de près par la collaboration avec les collègues et ensuite par la reconnaissance des élèves et la complicité avec eux. La collaboration est surtout envisagée comme un partage entre collègues ou encore entre amis. Le mentorat est aussi fortement prisé pour plusieurs d'entre eux. Ces éléments sont tous reliés aux facteurs humains et donc relationnels du métier.

Ma $2^{e}$ année j'ai changé d'école. J'étais choisie pour être la femme à tout faire - j'enseignais, $M / J$ - Éducation physique, $1^{\text {ère }}$ à la $6^{e}-$ Sciences et techno et $4^{e}$ à la $6^{e}$ année Anglais. C'était tout un apprentissage. Je me souviens encore de la $1^{\text {ère }}$ fois que je suis allée visiter l'école. Il y avait une autre enseignante qui préparait sa classe. Son sourire accueillant m'a marquée cette journée... Elle est devenue une collègue et une bonne amie avec qui j'ai partagé beaucoup de moments petits et grands.

Pour les enseignants du secondaire, la collaboration passe au premier plan. Celle-ci se vit au quotidien sur le plan de l'entraide et peut aussi prendre la forme de mentorat pour permettre de consolider les bases partiellement acquises. Les élèves sont également au cœur de leurs préoccupations, et semblent représenter un élément clé dans leur persévérance. Une dimension nouvelle s'ajoute, puisque les élèves n'apportent pas simplement un bien-être par le fait que «leurs yeux s'allument» mais aussi par la reconnaissance qu'ils manifestent à leurs enseignants. Enfin, ils précisent également que la persévérance vient aussi de leurs propres valeurs et des implications qu'elles engendrent. Ils parlent de l'enseignement comme d'un travail vivant et varié, au sein duquel ils apprennent tout le temps et font des projets, éléments se rattachant à ce que Bandura (1986) nomme les effets physiologiques et émotionnels.

C'est ce que je dis aux élèves j'enseigne parce que je continue d'apprendre, c'est la priorité. Je ne suis pas payé pour apprendre mais c'est un moyen détourné pour être comme ça. Je ne fais jamais deux fois le même cours, je n'enseigne pas souvent les mêmes ouvres. 
Soulignons que deux personnes affirment que la reconnaissance des parents a facilité leur persévérance. Notons que très peu d'enseignants ont soulevé cet élément comme étant un facteur de persévérance.

Pour les femmes (primaire et secondaire), les éléments à caractère humain sont les plus percutants, notamment en ce qui concerne les élèves en classe (les yeux qui s'allument) et la collaboration avec les collègues. Chez les hommes du niveau secondaire, les éléments reliés à la persévérance diffèrent un peu. Ils l'associent d'abord à la reconnaissance des élèves et ils mentionnent également le fait d'apprendre tout le temps, de monter des projets, etc).

c. Les années de diversification et de remise en questions.

Ce troisième groupe de onze enseignants (entre 11 et 20 ans d'expérience), a une représentation des facteurs de persévérance assez semblable à celle des enseignants de 4 à 10 ans, sauf peut-être pour quelques aspects qui seront discutés.

Les deux participants du secondaire sont totalement divergents dans leurs façons de se représenter les facteurs de persévérance, ce qui rend difficile l'élaboration d'une forme de pattern. Le premier enseignant possédant 20 ans d'expérience, accorde presque exclusivement de l'importance à la reconnaissance des élèves. La seconde, une enseignante ayant 11 ans d'expérience, insiste sur la mise sur pied de projets avec les élèves. Il faudra conséquemment rencontrer d'autres enseignants de niveau secondaire pour pouvoir faire émerger des patterns significatifs.

Pour ce qui est des enseignants du primaire, les deux éléments encore une fois les plus ciblés, sont la collaboration avec les collègues et les yeux qui s'allument. Ces enseignants relatent les moments marquants où ils ont été mentors et ont aidé d'autres enseignants novices ou des stagiaires, soit par une attitude de complicité, ou encore par l'échange. Ces personnes ont à cœur la formation par les pairs, et cette dimension professionnelle leur apparaît non seulement nécessaire, mais en plus, elle représente un atout pour persévérer et se réinvestir dans la profession, voire changer la routine.

Tableau 4.

Synthèse des analyses des entretiens et des histoires, pour les 11 à 20 ans d'expérience.

\begin{tabular}{|l|l|c|}
\hline Années d'expérience & Éléments récurrents (du + au -) & Nb. d'extraits \\
\hline Entre 11 et 20 ans & Collaboration avec les collègues & 8 \\
(primaire) & Les yeux qui s’allument & 7 \\
& Faire la différence & 5 \\
& Complicité & 5 \\
& Reconnaissance des élèves & 4 \\
& Vivant et varié & 4 \\
& Reconnaissance des parents & 2 \\
\hline Entre 11 et 20 ans & Direction & \\
(secondaire) & Seules deux personnes- propos contradictoires & \\
\hline
\end{tabular}




\begin{tabular}{|c|c|c|}
\hline $\begin{array}{l}\text { Entre } 11 \text { et } 20 \text { ans } \\
\text { (femmes) }\end{array}$ & $\begin{array}{l}\text { Les yeux qui s’allument } \\
\text { Collaboration avec les collègues } \\
\text { Faire la différence } \\
\text { Complicité } \\
\text { Reconnaissance des élèves } \\
\text { Vivant et varié } \\
\text { Reconnaissance des parents } \\
\text { Projets }\end{array}$ & $\begin{array}{l}6 \\
6 \\
6 \\
6 \\
4 \\
3 \\
3 \\
2\end{array}$ \\
\hline $\begin{array}{l}\text { Entre } 11 \text { et } 20 \text { ans } \\
\text { (hommes) }\end{array}$ & $\begin{array}{l}\text { Collaboration avec les collègues } \\
\text { Les yeux qui s’allument } \\
\text { Reconnaissance des élèves } \\
\text { Vivant et varié } \\
\text { Reconnaissance des parents }\end{array}$ & $\begin{array}{l}3 \\
2 \\
2 \\
2 \\
2\end{array}$ \\
\hline
\end{tabular}

En ce qui a trait à la compréhension des savoirs par les élèves, ces enseignants mettent l'accent sur la réussite de ces derniers et sur le fait qu'ils se relèvent de leurs difficultés. Néanmoins pour ces enseignants, la persévérance dans le métier est vue comme quelque chose qui va de soi parce qu'ils aiment cette profession.

En salle de classe, c'est le progrès que l'enfant fait, quand ça débloque, quand ils comprennent un concept, c'est simple, mais c'est ça.

Mais, pour moi, l'éducation, j'aime ça! Faire progresser un enfant, le voir grandir, c'est un petit pas en avant et je n'ai jamais pensé de quitter l'enseignement.

C'est exclusivement à ce niveau qu'apparaît de manière plus spécifique, l'agentivité traduite ici comme le fait de pouvoir faire la différence auprès des élèves. Cette dimension retrouvée dans quelques verbatim des participants, réapparaît ici dans 5 extraits qui y font explicitement référence. Ces professionnels ayant entre 11 et 20 d'expérience et qui se situent dans la phase professionnelle de «diversification», semblent s'accomplir professionnellement en raison du fait qu'ils croient «être utiles pour la société et qu'ils peuvent contribuer à changer le monde, un à la fois».

Je ne peux relire ces mots sans penser à sa tête blonde et échevelée comme celle du petit prince. De bien des façons, ces mots résument un des meilleurs souvenirs de ma carrière et me rends fière d'avoir pu faire la différence pour cet élève. Ce qui m'a incitée à rester dans l'enseignement, c'est certainement de penser que je pouvais faire la différence dans la vie d'enfants, en donnant le meilleur de moi-même; d'avoir l'impression de bâtir avec eux mais également d'avoir pu avoir une influence sur le cours des choses dont les approches pédagogiques utilisées.

Un autre trait particulièrement caractéristique de ce groupe se traduit par le fait qu'il est le seul à parler de manière spécifique et en grand nombre (5 extraits), de la complicité entre les élèves et face à eux. L'occasion aussi d'encourager les jeunes à faire preuve d'humour et à adopter une attitude positive face à l'apprentissage, face aux autres et plus généralement, face à la vie. 
Enfin, la reconnaissance des élèves, des parents et de la direction, sont aussi des facteurs déterminants dans leur vécu professionnel. Ici encore, les enseignants de cette phase mentionnent en plus grand nombre, l'importance de la reconnaissance des parents, et ils sont les seuls à aborder la direction comme un facteur de persévérance.

d. Les années de sérénité et de conservatisme

Finalement, en ce qui concerne les enseignants de 21 à 30 ans d'expérience, il s'agit de cinq enseignants près de leur retraite ou déjà à la préretraite. Quatre d'entre eux oeuvrent au secondaire. Chez ces enseignants, la persévérance durant toutes ces années, s'explique par leur travail au quotidien auprès des élèves et par le fait qu'ils apprennent tout le temps. L'enseignante du primaire met pour sa part, également l'emphase sur le bonheur de concevoir des projets avec les élèves.

Les élèves de classes régulières avec leur passion des découvertes m'ont fait grandir et m'ont inspirée. Nous avons réalisé mille et un projets, apprivoisant Internet qui faisait timidement ses pas dans le monde scolaire. En découvrant la vraie vie dans la salle de classe, je voyais mes élèves s'épanouir. C'était pour moi un véritable paradis où chaque matin, je retrouvais la magie de la forêt enchantée des projets que nous bâtissions ensemble. J'y apprenais la fragilité des uns, je découvrais mes doutes et je me surprenais à une écoute chaque jour plus attentive de ce qui m'entourait et des êtres qui finalement dépendaient de moi. Leur flamme d'apprendre était ardente et j'adorais ravitailler ces êtres lumineux qui avaient tellement soif de découvertes.

\section{Les divergences au travers les histoires}

Cette section présente un portrait des éléments soulevés par certains enseignants, et non relevés par d'autres.

On remarque que les éléments non mentionnés ou ciblés une seule fois, varient entre les novices et les plus expérimentés. Le fait que les novices occultent en bonne partie des éléments, tels la reconnaissance des élèves et de la direction, la complicité avec les élèves ou le fait de faire la différence, trouve probablement sa source dans leur inexpérience dans le métier. En ce qui concerne la mise en oeuvre de projets avec les élèves qui n'est pas mentionnée et ce, indépendamment du fait que la formation initiale leur ait permis d'expérimenter de telles situations, on peut penser à l'instar de Perrenoud (1996), que cette conjoncture s'explique par le fait que le nouvel enseignant est : " "entre deux chaises ", hésitant entre les modèles reçus durant la formation initiale et les recettes plus pragmatiques qui ont cours dans le milieu professionnel ».

Pour les enseignants de 4 à 10 ans et de 11 à 20 ans d'expérience, on constate d'emblée qu'ils se sentent solides et que leurs compétences semblent bien développées et consolidées. Effectivement, ils mentionnent peu ou prou le fait d'apprendre tout le temps, que l'enseignement est vivant et varié ou encore la question de la reconnaissance des élèves, des parents ou de la direction. On sent 
cependant que cette consolidation n'inclut pas chez eux les projets pédagogiques à réaliser avec les élèves, la complicité à développer avec eux (surtout au secondaire) ni le fait qu'ils puissent faire une différence. Toutefois ces enseignants persistent, démontrent de l'enthousiasme et surtout, si l'on en juge les premiers tableaux, apprécient notamment le fait que les élèves réussissent et la collaboration avec les collègues.

Tableau 5.

Éléments non mentionnés et mentionnés qu’une seule fois par niveau.

\begin{tabular}{|l|l|l|}
\hline Années d'expérience & $\begin{array}{l}\text { Éléments mentionnés qu'une } \\
\text { seule fois }\end{array}$ & Éléments non mentionnés \\
\hline Entre 1 et 3 ans & $\begin{array}{l}\text { Reconnaissance des élèves } \\
\text { Reconnaissance des parents } \\
\text { Faire la différence } \\
\text { Complicité }\end{array}$ & $\begin{array}{l}\text { La direction } \\
\text { Les projets }\end{array}$ \\
\hline Entre 4 et 10 ans & $\begin{array}{l}\text { J'apprends tout le temps } \\
\text { Faire la différence } \\
\text { La direction }\end{array}$ & $\begin{array}{l}\text { Vivant et varié; } \\
\text { Reconnaissance des parents; } \\
\text { Les projets } \\
\text { Complicité }\end{array}$ \\
\hline Entre 11 et 20 ans & $\begin{array}{l}\text { Projets } \\
\text { Yeux qui s'allument } \\
\text { Reconnaissance des élèves } \\
\text { Vivant et varié } \\
\text { Collaboration avec collègues } \\
\text { Reconnaissance des parents }\end{array}$ & $\begin{array}{l}\text { Faire la différence } \\
\text { La direction } \\
\text { Complicité } \\
\text { J'apprends tout le temps }\end{array}$ \\
\hline
\end{tabular}

À la lecture de ces données, on constate que certains éléments privilégiés par les enseignants, semblent absents de la littérature sur la persévérance. Notons particulièrement des éléments d'ordre extrinsèque, tels la reconnaissance des élèves, des parents ou de la direction ou encore, des éléments touchant le contexte de travail (vivant et varié). La poursuite de cette recherche permettra donc de mieux étayer ce concept de persévérance en y incluant ces dimensions. En dernière partie, il sera question d'un retour sur ces éléments en vue de permettre l'émergence d'une liste de pistes potentielles de travail, susceptibles d'aider les novices en enseignement.

\section{Vers une liste de pistes possibles en insertion professionnelle}

Une liste argumentée et relativement exhaustive de ces dispositifs, allant des plus importants aux moins importants, a été élaborée. Trois groupes de discussion ont eu lieu pour valider cette liste et permettre ainsi d'expliquer l'importance de la mise en place de ces dispositifs.

\section{Instituer le mentorat}

Le travail avec les autres collègues est perçu comme essentiel chez la plupart des participants à la recherche. Cité en parallèle avec «les yeux qui s'allument», élément révélateur du travail enseignant au quotidien, la 
collaboration entre collègues, associée dans une certaine mesure à un sentiment d'appartenance à un groupe, est fort révélatrice des besoins de partage et de soutien de la part des enseignants. Le mentorat apparaît donc comme une des sources majeures de persévérance. Ce type d'entraide existe actuellement de manière informelle, puisque plusieurs participants ont relaté des expériences à l'intérieur desquelles ils ont été aidés par des collègues. Il semble que les novices apprécient un mentor souple, présent, relativement au fait des nouveautés et de la réforme, accompagnateur et par conséquent, guide. Les instances soucieuses d'instaurer des programmes de mentorat pour les novices, devraient ainsi considérer la sélection des personnes pouvant être mentors et ensuite, leur offrir une formation adéquate dans l'accompagnement et la guidance d'un novice. Ce dernier cherche effectivement à apprendre le métier, à vivre intensément chaque instant et à survivre dans la salle de classe (tous des éléments très prisés par les participants ayant entre 1 à 3 ans d'expérience). Le mentor doit ainsi, accompagner ce novice dans sa quête d'informations et répondre à ses besoins immédiats, tout en lui permettant d'évoluer dans l'acquisition de nouvelles habiletés et dans le développement de ses compétences professionnelles. Il doit encourager l'innovation, sans toutefois y trop insister. Il s'agit d'une méthode dynamique d'accompagnement des sujets qui favorise le dialogue (qui) permet de rendre explicite, d'ajuster, de modifier ce que les sujets pensent d'une situation de laquelle ils désirent apprendre. (Phillion, 2005, p. 76). Bandura (1986), abonde en ce sens lorsqu'il explique en quoi les expériences vicariantes favorisent le sentiment d'efficacité des personnes et entraînent conséquemment la persévérance. Ce principe de modelage, de regard sur l'autre et avec l'autre, et de réflexion sur sa pratique ne peut qu'être bénéfique pour le novice au prise avec une situation aussi complexe que la salle de classe avec laquelle il doit «faire avec», jour après jour.

\section{Prévoir des formations continues}

Plusieurs novices ont mentionné que les formations offertes par le conseil scolaire, leur avaient été bénéfiques. Il semble donc judicieux de maintenir ces sessions à contenu informatif ou technique, ou encore axées sur certaines dimensions du curriculum, pour justement permettre à ces nouveaux enseignants de se familiariser avec leur nouvel environnement. Par contre, il importe de ne pas se limiter à la seule offre de structures de ce type, mais également de favoriser la motivation intrinsèque envers l'accomplissement et le perfectionnement, par des formations centrées, à la fois sur les besoins spécifiques des débutants, et axées sur des pistes de réflexion pour favoriser leur évolution dans le métier (Ryan et Deci 2000). De telles formations continues travaillent entre autres, sur le processus de socialisation à la profession enseignante, sur une meilleure insertion dans un monde qui a un fonctionnement qui lui est propre, ainsi que sur l'éclosion d'un moi professionnel dans les premiers mois du vécu en salle de classe (Hétu, Lavoie et Baillauques, 1999). Ces formations axées sur le partage entre novices, et guidées par des spécialistes (conseillers pédagogiques) peuvent alors favoriser le développement de l'identité professionnelle laquelle est susceptible d'aider à la persévérance dans le métier (Lebel, 2005). 


\section{Impliquer la direction}

Le support et l'appui de la direction est un facteur peu relevé par les participants. Bien que certains y fassent allusion, la plupart mettent en doute l'importance de la direction comme facteur de persévérance. Pourtant, les quelques personnes qui évoquent cet élément sont convaincues que «si la direction n'avait pas été là, ils n'y seraient plus». Ceci nous amène à supposer que si les participants ont peu mentionné le support de la direction, cela peut être attribuable au fait qu'ils n'ont pas connu de nombreuses expériences en matière de support de la part des directions et donc qu'ils voient difficilement comment « une direction qui a autre chose à faire» peut avoir le temps d'aider les nouveaux enseignants. À cet égard, Gather-Thurler (2000, p. 153) relevant des recherches sur les écoles efficaces et innovantes, mentionne "qu'aucun changement important ne peut se réaliser sans l'intervention de quelques leaders affirmés et reconnus». Sur le plan de la persévérance, la direction pourrait entre autres, accentuer l'écoute pour accueillir les états physiologiques de la personne (Bandura, 1986). Pour ce faire, même si ces éléments n’ont pas de valeur scientifique en tant que tel, la direction pourrait instaurer un leadership afin de créer des situations et des projets suscitant l'enthousiasme et la passion, afin de faciliter l'émergence de l'autoefficacité en aidant les novices à gérer et à organiser leurs multiples tâches et en les engageant dans des projets qui les tiennent à cœur, sans toutefois les y obliger.

\section{Se prendre en mains}

Au-delà de ces quelques pistes d'accompagnement des novices, il demeure qu'ils sont eux-mêmes les premiers instigateurs de leur insertion et réussite professionnelles. Ils doivent cependant avoir l'impression qu'ils ont le contrôle de leurs gestes et de leurs actions pour pouvoir développer un sentiment d'efficacité personnelle suffisamment fort pour les aider à persévérer dans des moments plus difficiles. À cet égard, Perrenoud (2001) relève les besoins des débutants en ce qui a trait au savoir-analyser dans diverses situations ne touchant pas uniquement les élèves et la classe, mais également entre collègues. Ce travail sur soi implique aussi l'étude de problèmes complexes, l'analyse du changement, l'apprentissage à «l'agir dans l'urgence» et au savoir évoluer. Ces divers besoins nécessitent donc un travail sur soi et sur le sens de la profession par l'émergence en formation initiale et continue, de situations d'accompagnement et de médiations susceptibles d'alimenter la passion pour l'exercice du métier et le besoin de toujours apprendre.

\section{Notes:}

1. Cette recherche a été possible grâce à une subvention du Ministère de l'Éducation de l'Ontario pour l'année 2004-2005. Elle a été possible aussi grâce à la participation de Madame Diane Démoré de l'Université Laurentienne et du travail d'assistanat de recherche de Ann-Louise Davidson.

2. Ce nombre se chiffre présentement dans les 175 . 
3. Selon l'Ordre des enseignantes et des enseignants de l'Ontario (2003), ce taux grimpe parfois jusqu’à $50 \%$ certaines années.

\section{Références}

Argyle, M. (1997). L'effet des variables environnementales sur le bonheur. Revue québécoise de psychologie, 18(2), 75-98. Traduit et adapté de l'anglais par Benoît Giroux.

Bandura, A. (1986). Social foundations of thought and action: A social cognitive theory. Englewood-Cliffs, N.J.: Prentice-Hall.

Bandura, A. (2001). Social Cognitive Theory: And Agentic. Annual Review of Psychology, 54(1), 1-26.

Bandura, A. (2003). Autoefficacité. Le sentiment d'efficacité personnelle. Paris : De Boeck. Traduite de l'anglais : 1997 par J. Lecomte. Self-efficacy. New York : Freeman \& Co.

Bourdages, L. (2001). La persistance aux études supérieures, le cas du doctorat. SainteFoy, PUQ.

Csikszentmihalyi, M. (1990). Flow: The psychology of optimal experience. New York, Harper \& Row.

Cyrulnik, B. (1999). Un merveilleux malheur. Paris, Odile Jacob.

Deci, E.L. \& Ryan, R.M. (1985). Intrinsic motivation and self-determination in human behavior. New York, Plenum.

Dubé, L., Kairouz, S. \& Jodoin, M. (1997). L'engagement: Un gage de bonheur? Revue québécoise de psychologie, 18(2) 1997, 211-237.

Festinger L. (1964). Conflict, decision and dissonance. Stanford, Stanford University Press.

Gather Thurler, M. (2000). Innover au cœur de l'établissement scolaire, Paris : ESF.

Hebb, D. O. (1949). The Organization of behavior: A neuropsychological theory. New York, Wiley.

Hétu, J.-C., Lavoie, M., et Baillauques, S. (1999) Jeunes enseignants et insertion professionnelle, Bruxelles : De Boeck.

Heutte, J. (2006). Blocs-notes de Jean Heude, http://jean.heutte.free.fr/article.php, consulté en avril 2007.

Huberman, M. (1989). La vie des enseignants : évolution et bilan d'un profession, Lausanne : Delachaux et Niestlé.

Kiesler, C. (1971). The psychology of commitment. New-York, New York Academic Press.

Lani-Bayle, M. \& Bire, N. (2003). Une pratique : les histoires de vie, Université de Nantes. www.frjp.com/lani-bayle/pages/Histoire vie2.htm

Lebel, C. (2005). Persévérance et identité professionnelle, Communication à l'ACFAS dans le cadre du colloque : persévérance en enseignement.

Legendre, R. (1993). Dictionnaire actuel de l'éducation, 2e éd. Montréal, Guérin.

Lessard, C. \& Tardif, M. (2003). Les identités enseignantes (1960-1990). Sherbrooke: CRP

Malrieu, Ph. (2003).La construction du sens dans les dires autobiographiques, Paris : Érès. Montclerc, G.-J. (2006). Pour une éthique de l'autoformation, Bulletin des APP, 66, Paris. 
Mukamurera (2006). Le décrochage des enseignants. Il faut sonner l'alarme. Texte de l'entretien intégral réalisé par Caron. L., avec Mukamurera. J. Récupérée au http://cbcsq.qc.net/sites/1673/documents/secteurs/entrevue_j_moukamouderas.pdf, le 29 Mars, 2006.

Myers, D et Diener, E. (1997). La poursuite scientifique du bonheur. Revue québécoise de psychologie, 18(2) 1997, 13-28. Traduit et adapté de l'anglais par Léandre Bouffard.

Olivier, G. et Demouge, N. (1999). L'enquête orale, L'histoire de vie : une approche phénoménologique de la recherche, Spirale, 23, 195-212.

Ordre des enseignants et des enseignantes de l'Ontario, (2003a). Transition à l'enseignement, rapport des nouveaux enseignants de 2001-2002 sur leurs deux premières années d'enseignement en Ontario. www.oct. ca/fr/CollegePublications.

Ordre des enseignants et des enseignantes de l'Ontario, (2003b). Insertion professionnelle des nouveaux enseignants et enseignantes : Grandir dans la profession. www.oct.ca/fr/CollegePublications/news_archive/20030411_f.asp .

Perrenoud, Ph. (2001). Préparer les enseignants au changement, Texte de la communication aux:Journées Éducation et Prospective, Forum de l'innovation, Suissse: Ollioules.

Perrenoud, Ph. (1996). Former des enseignants débutants qui deviendront des praticiens réflexifs. Forum-Pédagogies (Bruxelles), Janvier, pp. 10-12

Phillion, R. (2005) Prise en compte des représentations des étudiants mentors au regard de leur pratique et de leurs besoins en terme de formation, thèse de doctorat, Université d'Ottawa.

Rayou, P. et Van Zanten. A. (2004). Enquête sur les nouveaux enseignants ; changeront-ils l'école ?, Paris :

Ryan, R. M. \& Deci, E. L. (2000). Self-determination theory and the facilitation of intrinsic motivation, social development, and well-being. American Psychologist, 55(1), 68-78.

Secord, P. F. \& Backman, C. W. (1974). Social Psychology . New Jersey, McGraw-Hill.

Singer, R, Hausenblas, H, Janelle, C. (2001). Handbook of sport psychology, $2^{\mathrm{e}}$ éd. John Wiley \& Sons, Inc, Toronto.

Louise M. Bélair, Ph.D. en mesure et évaluation, est professeure au département des sciences de l'éducation de l'université du Québec à Trois-Rivières et directrice du programme BEPEP. Elle est membre du groupe MARDIF (maîtrise de recherche à distance internationale francophone) chapeautée par l’Université de Rouen. Outre l'évaluation, ses divers champs d'intérêt touchent particulièrement la personne enseignante, son évolution, ses compétences et les facteurs permettant de favoriser sa persévérance. louise.belair@uqtr.ca

Christine Lebel, Ph.D. est professeure en formation pratique et responsable pédagogique des stages du préscolaire et primaire au département des sciences de l'éducation de l'université du Québec à Trois-Rivières. Elle s'intéresse à la formation initiale et continue des enseignants et particulièrement aux dispositifs et démarches de formation susceptibles d'intensifier la professionnalisation du métier. Sa recherche actuelle porte sur la démarche d'analyse de construits au service de la persévérance des enseignants. christine.lebel@uqtr.ca 